\title{
Mild and highly chemoselective oxidation of thioethers mediated by $\operatorname{Sc}(\text { OTf })_{3}$
}

\author{
Mizio Matteucci, ${ }^{\dagger}$ Gurdip Bhalay ${ }^{\ddagger}$ and Mark Bradley ${ }^{\dagger}$ \\ ${ }^{\dagger}$ Combinatorial Centre of Excellence, Department of Chemistry, University of Southampton, Southampton \\ SO17 1BJ, United Kingdom and "Novartis Pharma, Wimblehurst Road, Horsham RH12 5AB, United \\ Kingdom
}

\section{Supporting Information}

General informations: Phenyl methyl sulfide, $p$-bromophenyl methyl sulfide, $p$ methoxyphenyl methyl sulfide and 1-cyclohexyl-3-(2-morpholino-ethyl)carbodiimide metho- $p$-toluenesulfonate were purchased from Aldrich. Sulfones $3 a^{1}, 3 b^{2}, 3 c^{3}, 3 d^{3,4}$ are described in the literature. Scandium(III) trifluoromethanesulfonate hydrate was purchased by Lancaster. Amino acids and Fmoc-OSu were purchased from Novabiochem. Amino acids are abbreviated using the standard-3-letter code and are of configuration $\mathrm{L}$. $\mathrm{H}_{2} \mathrm{O}_{2}$ was used as a $60 \%$ solution in water $\left(d=1.19 \mathrm{~g} \mathrm{~mL}^{-1}\right)$, supplied by Fisher. $\mathrm{CH}_{2} \mathrm{Cl}_{2}$ was distilled from calcium hydride and stored over Type $3 \mathrm{~A}$ molecular sieves. GC were run in a GC Varian 3300/3400 equipped with a flame ionization detector and a capillary column (DBWAX) , 30mx0.53mm. Run conditions: initial temperature: $50 \mathrm{C}$ for 2 minutes; temperature increase rate: $20 \mathrm{C} / \mathrm{min}$; final temperature: $220 \mathrm{C}$. GCCIMS were run on a ThermoQuest TraceMS gas chromatography mass spectrometer, ionisation technique: chemical ionisation. RP-HPLC analysis were carried out with a Hewlett Packard Chemstation HP1100 Chemstation. Conditions 1) Column- $\mathrm{C}_{18}$ ODS, i.d. $150 \mathrm{~mm} \times 3 \mathrm{~mm}$ (Analytical, flow $0.5 \mathrm{mLmin}^{-1}$ ). Gradient: $0.1 \% \mathrm{TFA} / \mathrm{H}_{2} \mathrm{O}$ to $0.1 \%$ TFA/MeCN over $20 \mathrm{~min}$. Conditions 2) Column- $\mathrm{C}_{18}$ ODS, i.d. $4.6 \mathrm{~mm} \mathrm{x} 3 \mathrm{~mm}$ (Analytical, flow $1 \mathrm{mLmin}^{-1}$ ). Gradient: $0.1 \% \mathrm{TFA} / \mathrm{H}_{2} \mathrm{O}$ to $0.1 \% \mathrm{TFA} / \mathrm{MeCN}$ over 5 minutes. Detection by UV (220 and $254 \mathrm{~nm}$ ) and ELS detectors. ${ }^{1} \mathrm{H}$ NMR spectra were recorded on Bruker AC300 or Bruker DPX $400 \mathrm{MHz}$ spectrometers, ${ }^{13} \mathrm{C}$ NMR spectra were recorded on Bruker AC300 (75 MHz) or Bruker DPX $400 \mathrm{MHz}(100 \mathrm{MHz})$ spectrometers and reported in units of $(\mathrm{ppm})$ relative to solvents $\left(\mathrm{CDCl}_{3}\right.$ 7.27, 
DMSO- $\mathrm{d}_{6} \quad 2.50, \mathrm{D}_{2} \mathrm{O} \quad 4.79$ for ${ }^{1} \mathrm{H}$ NMR and $\mathrm{CDCl}_{3} \quad$ 77.16, DMSO- $\mathrm{d}_{6} \quad 40.45$ for ${ }^{13} \mathrm{C}$ NMR). ${ }^{45} \mathrm{Sc}$ NMR spectra were recorded on Bruker DPX $400 \mathrm{MHz}$ (97 MHz) spectrometer and are reported in ppm using $\mathrm{Sc}\left(\mathrm{NO}_{3}\right)_{3}$ in $\mathrm{D}_{2} \mathrm{O} / \mathrm{H}_{2} \mathrm{O}$ as external standard ( 0.0). ES mass spectra were recorded by using a VG Platform Quadrupole Electrospray Ionisation mass spectrometer, measuring monoisotopic masses. HRMS were run on a Bruker APEX3. Infra-red spectra were recorded on a BIORAD Golden Gate FTS 135. All samples were run neat as solids or liquids. Melting points were determined using a Gallenkamp melting point apparatus and are uncorrected.

Abbreviations: CMC: 1-cyclohexyl-3-(2-morpholino-ethyl)carbodiimide metho- $p$ toluenesulfonate; HMPA: hydroxymethylphenoxyacetic acid; HOBt: 1hydroxybenzotriazole; NMM: $N$-methyl morpholine; $\operatorname{Sc}(\mathrm{OTf})_{3}$ : scandium trifluoromethanesulfonate; TFA: trifluoroacetic acid; TIS: triisopropyl silane.

\section{Synthesis of thioethers.}

Phenyl prenyl sulfide (la). ${ }^{5}$ Yield: 83\%; $R_{f}$. 0.32 (200/1, hexanes/EtOAc); ${ }^{1} \mathrm{H}-\mathrm{NMR}$ $\left(\mathrm{CDCl}_{3}, 400 \mathrm{MHz}\right): \quad 7.35-7.13(\mathrm{~m}, 5 \mathrm{H}), 5.30(\mathrm{~m}, 1 \mathrm{H}), 3.53(\mathrm{~d}, 2 \mathrm{H}, J=8 \mathrm{~Hz}), 1.70(\mathrm{~s}$, 3H), 1.58 (s, $3 \mathrm{H}) ;{ }^{13} \mathrm{C}-\mathrm{NMR}\left(\mathrm{CDCl}_{3}, 100.6 \mathrm{MHz}\right): \quad 137.2,136.6,130.0,129.0,126.2$, 119.7, 32.5, 25.9, 18.0; GC-CIMS: rt $10.78 \mathrm{~min}, \mathrm{~m} / \mathrm{z}: 179(\mathrm{M}+\mathrm{H})^{+}$; HPLC ( 254$): 13.5$ $\min$ (conditions 1).

$\mathrm{H}$-Cys(Me)-OMe $\mathrm{HCl}^{6}{ }^{6}$ An adapted literature protocol for the formation of amino acid methyl esters was used. ${ }^{7} \mathrm{H}-\mathrm{Cys}(\mathrm{Me})-\mathrm{OH}(5 \mathrm{~g}, 37 \mathrm{mmol}$ ) was suspended in 2,2dimethoxypropane $(150 \mathrm{~mL})$ and conc. hydrochloric acid $(15 \mathrm{~mL})$ added. The reaction was stirred overnight. The mixture was concentrated in vacuo; the residue, dissolved in a minimum amount of $\mathrm{MeOH}(25 \mathrm{~mL})$, was added dropwise to cold $\mathrm{Et}_{2} \mathrm{O}(500 \mathrm{~mL})$. The resulting white precipitate was collected by filtration and dried under vacuum.

mp: (145-146) C (lit. 143-144) $\mathrm{C}^{8} ;{ }^{1} \mathrm{H}-\mathrm{NMR}\left(\mathrm{CDCl}_{3}, 300 \mathrm{MHz}\right): 4.38$ (dd, $J=10,6$ $\mathrm{Hz}, 1 \mathrm{H}, H$ ), 3.83 (s, 3H, OCH $\left.H_{3}\right), 3.17$ (dd, $J=20,6 \mathrm{~Hz}, 1 \mathrm{H}, H \mathrm{H}_{2}$ ), $3.04(\mathrm{dd}, J=20,10$ $\left.\mathrm{Hz}, 1 \mathrm{H}, \mathrm{HH}_{2}\right), 2.12$ (s, 3H, SCH 3$) ;{ }^{13} \mathrm{C}-\mathrm{NMR}\left(\mathrm{CDCl}_{3}, 75.5 \mathrm{MHz}\right): \quad 172.0\left(\mathrm{CO}_{2} \mathrm{Me}\right)$, $56.4\left(\mathrm{OCH}_{3}\right), 54.4(\mathrm{C}), 36.0(\mathrm{C}), 17.3\left(\mathrm{SCH}_{3}\right)$.

Fmoc-Cys(Me)-OMe (4a). H-Cys(Me)-OMe $\mathrm{HCl}(1.25 \mathrm{~g}, 8.4 \mathrm{mmol}$, 1 equiv) was dissolved in $10 \% \mathrm{Na}_{2} \mathrm{CO}_{3}$ in water $(19 \mathrm{~mL})$. The solution was cooled to $0 \mathrm{C}$ in an ice 
bath and dioxane $(19 \mathrm{~mL})$ was added. Fmoc-OSu (3.40 g, $10 \mathrm{mmol}, 1.2$ equiv) was added and the solution allowed to warm to room temperature and stirred for 1 hour. The mixture was then diluted with water (to $c a .80 \mathrm{~mL}$ ) and extracted with EtOAc (2x100 mL). The extracts were dried $\left(\mathrm{Na}_{2} \mathrm{SO}_{4}\right)$, filtered and the solvent removed in vacuo. Column chromatography (silica gel, hexanes/EtOAc, 7/3) gave a white solid which was recrystallised from EtOH/EtOAc to afford the desired compound ( $2 \mathrm{~g}, 64 \%) . R_{f}: 0.50$ (hexanes/EtOAc); mp: (117-120) C; IR (neat): ${ }_{\max }: 3322,1754,1736,1685 \mathrm{~cm}^{-1} ;{ }^{1} \mathrm{H}-$ NMR $\left(\mathrm{CDCl}_{3}, 300 \mathrm{MHz}\right): 7.79 \& 7.61\left(2 \mathrm{~d}, J=7 \mathrm{~Hz}, 4 \mathrm{H}\right.$, Fmoc- $\left.H_{1,4,5,8}\right), 7.42 \& 7.33$ $\left(2 \mathrm{t}, J=7 \mathrm{~Hz}, 4 \mathrm{H}\right.$, Fmoc- $\left.H_{2,3,6,7}\right), 5.67$ (d, $\left.J=8 \mathrm{~Hz}, 1 \mathrm{H}, \mathrm{NH}\right), 4.70-4.60$ (m, 1H, Cys(Me)$H$ ), $4.43\left(\mathrm{~d}, J=7 \mathrm{~Hz}, 2 \mathrm{H}, \mathrm{Fmoc}-\mathrm{CH}_{2}\right), 4.25$ (t, $\left.J=7 \mathrm{~Hz}, 1 \mathrm{H}, \mathrm{Fmoc}-\mathrm{CH}\right), 3.80$ (s, 3H, $\left.\mathrm{OCH}_{3}\right), 2.99\left(\mathrm{~m}, 2 \mathrm{H}, \mathrm{Cys}(\mathrm{Me})-\mathrm{H}_{2}\right), 2.12$ (s, 3H, Cys(Me)-SCH$\left.{ }_{3}\right) ;{ }^{13} \mathrm{C}-\mathrm{NMR}\left(\mathrm{CDCl}_{3}\right.$, $75.5 \mathrm{MHz}): 171.5\left(\mathrm{CO}_{2} \mathrm{Me}\right), 155.8(\mathrm{OCONH}), 143.8$ (Fmoc- $\left.C_{8 \mathrm{a}, 9 \mathrm{a}}\right), 141.4$ (Fmoc$\left.C_{4 \mathrm{a}, 4 \mathrm{~b}}\right), 127.8\left(\right.$ Fmoc- $\left.C_{1,8}\right), 127.2\left(\right.$ Fmoc- $\left.C_{4,5}\right), 125.2\left(\right.$ Fmoc- $\left.C_{2,7}\right), 120.1$ (Fmoc- $\left.C_{3,6}\right), 67.3$ $\left(\mathrm{Fmoc}-\mathrm{CH}_{2}\right), 53.5$ (Cys(Me)-C ), $52.8\left(\mathrm{OCH}_{3}\right), 47.2$ (Fmoc- $\left.\mathrm{CH}\right), 36.8$ (Cys(Me)-C ), $16.4\left(\mathrm{Cys}(\mathrm{Me})-\mathrm{SCH}_{3}\right)$; ES-MS: $m / z: 394.2(\mathrm{M}+\mathrm{Na})^{+}, 765.4(2 \mathrm{M}+\mathrm{Na})^{+}$; HRMS (ES+): calcd for $\mathrm{C}_{20} \mathrm{H}_{21} \mathrm{NO}_{4} \mathrm{SNa}(\mathrm{M}+\mathrm{Na})^{+}$394.1090, found 394.1083; HPLC ( 254$): 12.4$ min (conditions 1).

H-Cys(Trt)-OMe HCl. ${ }^{9}$ Yield: 98\%; $R_{f}: 0.60\left(\mathrm{CHCl}_{3} / 5 \% \mathrm{MeOH}\right) ; \mathrm{mp}:(70-75) \mathrm{C}$ (lit. $78 \mathrm{C}$ ); IR (neat): $\quad \max _{\max } 1743 \mathrm{~cm}^{-1} ;{ }^{1} \mathrm{H}-\mathrm{NMR}$ (DMSO-d $6,400 \mathrm{MHz}$ ): 8.90 (br s, 3H, $\left.\mathrm{NH}_{3}{ }^{+}\right), 7.42-7.20(\mathrm{~m}, 15 \mathrm{H}, \mathrm{ArH}), 3.83-3.75(\mathrm{~m}, 1 \mathrm{H}, H), 3.70\left(\mathrm{~s}, 3 \mathrm{H}, \mathrm{OCH}_{3}\right), 2.76(\mathrm{dd}$, $1 \mathrm{H}, J=13,6 \mathrm{~Hz}, H \mathrm{H}$ ), $2.63(\mathrm{dd}, 1 \mathrm{H}, J=13,6 \mathrm{~Hz}, \mathrm{H} H) ;{ }^{13} \mathrm{C}-\mathrm{NMR}\left(\mathrm{DMSO}_{6}, 100.6\right.$ $\mathrm{MHz}): \quad 169.0\left(\mathrm{CO}_{2} \mathrm{Me}\right), 144.5(\mathrm{Ar}-i p s o C), 129.9(\mathrm{ArCH}), 129.1(\mathrm{ArCH}), 127.9(\mathrm{ArCH})$, $67.5\left(\mathrm{Ar}_{3} C\right), 53.9\left(\mathrm{OCH}_{3}\right), 51.9(C), 32.2(C)$; ESMS: $m / z: 378.3(\mathrm{M}+\mathrm{H})^{+}, 755.6$ $(2 \mathrm{M}+\mathrm{H})^{+} ;$HPLC (ELS): 9.4 min (conditions 1).

Boc-Phe-Cys $(\mathrm{Me})-\mathrm{OH}$. Preparation according to a literature procedure. ${ }^{10}$ Yield: $93 \%$; $R_{f}$ : $0.62\left(\mathrm{CHCl}_{3} / 5 \% \mathrm{MeOH} / 1 \% \mathrm{AcOH}\right)$; mp: (43-50) C; IR (neat): ${ }_{\max }: 3303,1713,1643 \mathrm{~cm}^{-}$ ${ }^{1} ;{ }^{1} \mathrm{H}-\mathrm{NMR}$ (DMSO-d 6 , $400 \mathrm{MHz}$ ): 12.92 (br s, $\left.1 \mathrm{H}, \mathrm{CO}_{2} \mathrm{H}\right), 8.25$ (d, $1 \mathrm{H}, J=8 \mathrm{~Hz}$, PheNH), 7.39-7.18 (m, 5H, ArH), 6.93 (d, 1H, J = 9 Hz, Cys(Me)-NH), 4.49 (m, 1H, Phe$H$ ), 4.26 (m, 1H, Cys(Me)-H ), 3.04 (dd, 1H, $J=14,4$ Hz, Cys(Me)-HH ), 2.93 (dd, 1H, 
$J=14,5 \mathrm{~Hz}$, Phe- $H \mathrm{H}$ ), 2.84 (dd, 1H, $J=14,5 \mathrm{~Hz}$, Phe-HH ), 2.77 (dd, 1H, $J=14,11$ $\mathrm{Hz}, \mathrm{Cys}(\mathrm{Me})-\mathrm{H} H$ ), 2.14 (s, 3H, Cys(Me)-SCH$\left.{ }_{3}\right), 1.33$ (s, 9H, C(CH3) $) ;{ }^{13} \mathrm{C}-\mathrm{NMR}$ (DMSO-d 6 , $100.6 \mathrm{MHz}): \quad 172.9 \& 172.7\left(\mathrm{CO}_{2} \mathrm{Me} \& \mathrm{CONH}\right), 156.1(\mathrm{OCONH}), 139.0$ (ipso-ArC), $130.1 \& 128.9$ ( o \& m-ArC), 127.1 (p-ArC), $79.0\left(C\left(\mathrm{CH}_{3}\right)_{3}\right), 56.5(\mathrm{Cys}(\mathrm{Me})$ -

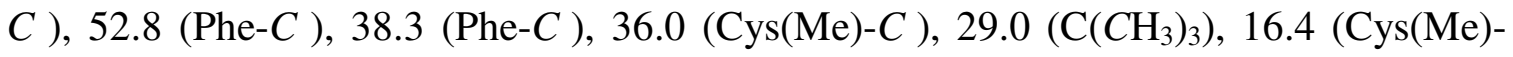
$\left.\mathrm{SCH}_{3}\right)$; ESMS: m/z: $381.3(\mathrm{M}-\mathrm{H})^{-}, 763.5(2 \mathrm{M}-\mathrm{H})^{-}$; HRMS $\left(\mathrm{ES}^{+}\right)$: calcd for $\mathrm{C}_{18} \mathrm{H}_{26} \mathrm{~N}_{2} \mathrm{O}_{5} \mathrm{SNa}(\mathrm{M}+\mathrm{Na})^{+}, 405.1454$, found 405.1458; HPLC (ELS): 9.9 min (conditions 1).

Boc-Phe-Cys(Me)-Cys(Trt)-OMe (4b). NMM (0.29 mL, $2.61 \mathrm{mmol}, 2$ equiv) was dissolved in dry $\mathrm{CH}_{2} \mathrm{Cl}_{2}(15 \mathrm{~mL})$. $\mathrm{H}$-Cys(Trt)-OMe $\mathrm{HCl}(0.84 \mathrm{~g}, 1.96 \mathrm{mmol}, 1.5$ equiv) in dry $\mathrm{CH}_{2} \mathrm{Cl}_{2}$ (30mL) was added followed by Boc-Phe-Cys(Me)-OH $(0.50 \mathrm{~g}, 1.31 \mathrm{mmol}, 1$ equiv) and $\mathrm{HOBt}(0.212 \mathrm{~g}, 1.57 \mathrm{mmol}, 1.2$ equiv). The mixture was cooled to $0 \mathrm{C}$ and CMC (0.83 g, $1.96 \mathrm{mmol}, 1.5$ equiv) added. The mixture was allowed to warm up to room temperature and left stirring for 3 hrs. It was then diluted with $\mathrm{CH}_{2} \mathrm{Cl}_{2}(18 \mathrm{~mL})$, washed with sat. $\mathrm{NaHCO}_{3}(20 \mathrm{~mL}), 1 \mathrm{M} \mathrm{HCl}\left(10 \mathrm{~mL}\right.$ x 3), aqueous $\mathrm{NaHCO}_{3}(5 \%, 10 \mathrm{~mL}$ x 3), brine $(10 \mathrm{~mL} \times 2)$, dried $\left(\mathrm{Na}_{2} \mathrm{SO}_{4}\right)$, filtered and the solvent removed in vacuo. Purification by column chromatography (silica gel, hexanes/EtOAc 4/6) gave the desired compound as a white foam $(0.63 \mathrm{~g}, 65 \%) . R_{f}: 0.54$ (hexanes/EtOAc); mp: (61-75) C; IR (neat): $\max : 3282,1735,1686,1643 \mathrm{~cm}^{-1} ;{ }^{1} \mathrm{H}-\mathrm{NMR}\left(\mathrm{CDCl}_{3}, 400 \mathrm{MHz}\right)$ : 7.28-7.01 (m, 20H, ArH), $6.76(\mathrm{~d}, 1 \mathrm{H}, J=7 \mathrm{~Hz}, \mathrm{NH}), 6.71(\mathrm{~d}, 1 \mathrm{H}, J=7 \mathrm{~Hz}, \mathrm{NH}), 4.79$ (br s, 1H, NH), 4.34-4.17 (m, 3H, $3 H$ ), 3.55 (s, 3H, OCH $H_{3}$ ), 2.94 (m, 2H, Phe- $H_{2}$ ), 2.73 (dd, $1 \mathrm{H}, J=14$, $4 \mathrm{~Hz}, \mathrm{Cys}(\mathrm{Me})-H \mathrm{H}$ ), 2.59-2.49 (m, 3H, Cys(Me)-HH \& Cys(Trt)- $\left.H_{2}\right), 1.98$ (s, 3H, Cys(Me)-SCH$\left.H_{3}\right), 1.25$ (s, 9H, C( $\left.\left.\mathrm{CH}_{3}\right)_{3}\right) ;{ }^{13} \mathrm{C}-\mathrm{NMR}\left(\mathrm{CDCl}_{3}, 100.6 \mathrm{MHz}\right): \quad 171.5,170.6$, $170.1\left(\mathrm{CO}_{2} \mathrm{Me} \& \mathrm{CONH}\right), 155.8(\mathrm{OCONH}), 144.6$ (Cys(Trt)-ipso-ArC), 136.7 (Phe-ipso$\operatorname{ArC})$, 129.9, 128.6, 129.1, 128.4, 127.4, $127.3(\mathrm{ArC}), 80.8\left(\mathrm{C}\left(\mathrm{CH}_{3}\right)_{3}\right), 67.4$ (Cys(Trt)$\left.\mathrm{Ar}_{3} \mathrm{C}\right), 53.0\left(\mathrm{OCH}_{3}\right), 52.2 \& 52.1$ (3C), 38.4 (Phe-C), 36.4 (Cys(Me)-C), 33.7

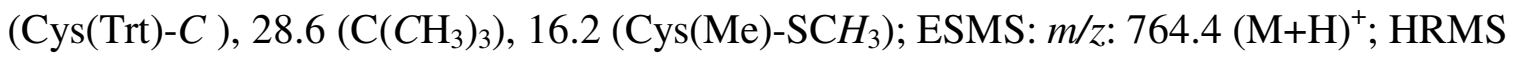
$\left(\mathrm{ES}^{+}\right.$): calcd for $\mathrm{C}_{41} \mathrm{H}_{47} \mathrm{~N}_{3} \mathrm{O}_{6} \mathrm{~S}_{2} \mathrm{Na}(\mathrm{M}+\mathrm{Na})^{+}, 764.2804$, found 764.2798; HPLC (ELS): 14.3 min (conditions 1 ). 
H-Ala-OAll (tosylate salt). ${ }^{11}$ Alanine ( $3 \mathrm{~g}, 33.7$ mmol, 1 equiv), allyl alcohol (22.9 mL, $33.7 \mathrm{mmol}, 1$ equiv) and $p$-toluene sulfonic acid ( $7.7 \mathrm{~g}, 40.4 \mathrm{mmol}, 1.2$ equiv) were refluxed in benzene $(270 \mathrm{~mL})$ under Dean and Stark conditions for 24 hours. The solution was evaporated to $20 \mathrm{~mL}$ and precipitated in cold $\mathrm{Et}_{2} \mathrm{O}$ to give a brownish powder $(8.5 \mathrm{~g}$, 89\%). $R_{f}: 0.33\left(\mathrm{CH}_{2} \mathrm{Cl}_{2} / 10 \% \mathrm{EtOH}\right) ; \mathrm{mp}:(79-80) \mathrm{C}$; IR (neat): $\max : 1774 \mathrm{~cm}^{-1} ;{ }^{1} \mathrm{H}-\mathrm{NMR}$ $\left(\mathrm{CDCl}_{3}, 400 \mathrm{MHz}\right): \quad 8.18\left(\mathrm{br} \mathrm{s}, 3 \mathrm{H}, \mathrm{NH}_{3}{ }^{+}\right), 7.73(\mathrm{~d}, 2 \mathrm{H}, J=8 \mathrm{~Hz}, \mathrm{Ar} H), 7.14(\mathrm{~d}, 2 \mathrm{H}, J=$ $8 \mathrm{~Hz}, \operatorname{Ar} H$ ), 5.79 (ddt, $1 \mathrm{H}, J=17,11,6 \mathrm{~Hz}, \mathrm{CH}=\mathrm{CH}_{2}$ ), 5.25 (dd, $1 \mathrm{H}, J=17,1 \mathrm{~Hz}$, $\left.\mathrm{CH}=\mathrm{CH} H_{\text {trans }}\right), 5.18\left(\mathrm{dd}, 1 \mathrm{H}, J=11,1 \mathrm{~Hz}, \mathrm{CH}=\mathrm{CH}_{\text {cis }} \mathrm{H}\right), 4.57(\mathrm{dd}, 1 \mathrm{H}, J=13,6 \mathrm{~Hz}$, $\left.\mathrm{C} H \mathrm{H}-\mathrm{CH}=\mathrm{CH}_{2}\right), 4.50\left(\mathrm{dd}, 1 \mathrm{H}, J=13,6 \mathrm{~Hz}, \mathrm{CH} H-\mathrm{CH}=\mathrm{CH}_{2}\right), 4.12-3.98(\mathrm{~m}, 1 \mathrm{H}$, Ala- $H$ ), 2.35 (s, $\left.3 \mathrm{H}, \mathrm{ArCH}_{3}\right), 1.47$ (d, $3 \mathrm{H}, J=7 \mathrm{~Hz}$, Ala- $\left.\mathrm{CH}_{3}\right) ;{ }^{13} \mathrm{C}-\mathrm{NMR}\left(\mathrm{CDCl}_{3}, 100.6 \mathrm{MHz}\right)$ :

$169.7\left(\mathrm{CO}_{2} \mathrm{All}\right), 141.3$ (ipso-ArC), 140.7 (ipso-ArC), $131.2\left(\mathrm{CH}=\mathrm{CH}_{2}\right), 129.0 \& 126.2(o$ \& $m-\mathrm{ArC}), 119.1\left(\mathrm{CH}=\mathrm{CH}_{2}\right), 66.8\left(\mathrm{CH}_{2}-\mathrm{CH}=\mathrm{CH}_{2}\right), 49.3(\mathrm{Ala}-\mathrm{C}), 21.5\left(\mathrm{ArCH}_{3}\right), 16.0$ (Ala- $\left.\mathrm{CH}_{3}\right)$; ESMS: $m / z: 170.9(\mathrm{M}+\mathrm{H}+\mathrm{MeCN})^{+}$.

Boc-Phe-Cys(Me)-Ala-OAll (4c). NMM (0.69 mL, $6.28 \mathrm{mmol}, 2$ equiv) was dissolved in dry $\mathrm{CH}_{2} \mathrm{Cl}_{2}(35 \mathrm{~mL})$. H-Ala-OAll (tosylate salt) $(1.42 \mathrm{~g}, 4.71 \mathrm{mmol}, 1.5$ equiv) in dry $\mathrm{CH}_{2} \mathrm{Cl}_{2}(70 \mathrm{~mL})$ was then added followed by Boc-Phe-Cys(Me)-OH (1.20 g, $3.14 \mathrm{mmol}$, 1 equiv) and $\mathrm{HOBt}(0.509 \mathrm{~g}, 3.76 \mathrm{mmol}, 1.2$ equiv). The mixture was then cooled to $0 \mathrm{C}$ and CMC (1.99 g, $4.71 \mathrm{mmol}, 1.5$ equiv) added and allowed to warm up to room temperature and left stirring for $3 \mathrm{hrs}$. The mixture was then diluted with $\mathrm{CH}_{2} \mathrm{Cl}_{2}(40$ $\mathrm{mL}$ ), washed with sat. $\mathrm{NaHCO}_{3}(45 \mathrm{~mL}), 1 \mathrm{M} \mathrm{HCl}\left(25 \mathrm{~mL}\right.$ x 3), aqueous $\mathrm{NaHCO}_{3}(5 \%$, $25 \mathrm{~mL} \times 3)$, brine $(25 \mathrm{~mL} \times 2)$, dried $\left(\mathrm{Na}_{2} \mathrm{SO}_{4}\right)$, filtered and the solvent removed in vacuo. Purification by precipitation from $\mathrm{CH}_{2} \mathrm{Cl}_{2} /$ hexanes afforded a white solid $(1.21 \mathrm{~g}$, 78\%). $R_{f}: 0.19$ ( $\mathrm{Et}_{2} \mathrm{O} / 30 \%$ hexanes); mp: (83-86) C; IR (neat): max: 3308, 1746, 1692, 1662, $1641 \mathrm{~cm}^{-1} ;{ }^{1} \mathrm{H}-\mathrm{NMR}\left(\mathrm{CDCl}_{3}, 400 \mathrm{MHz}\right): \quad 7.40-7.20$ (m, 5H, Phe-ArH), 6.98 (d, $1 \mathrm{H}, J=8 \mathrm{~Hz}, \mathrm{NH}), 5.96\left(\mathrm{ddt}, 1 \mathrm{H}, J=17,10,6 \mathrm{~Hz}, \mathrm{CH}=\mathrm{CH}_{2}\right), 5.38(\mathrm{dd}, 1 \mathrm{H}, J=17,1 \mathrm{~Hz}$, $\left.\mathrm{CH}=\mathrm{CH} H_{\text {trans }}\right), 5.30\left(\mathrm{dd}, 1 \mathrm{H}, J=10,1 \mathrm{~Hz}, \mathrm{CH}=\mathrm{CH}_{\text {cis }} \mathrm{H}\right), 5.02(\mathrm{~d}, 1 \mathrm{H}, J=7 \mathrm{~Hz}, \mathrm{NH})$, 4.75-4.63 (m, 2H, $\left.\mathrm{CH}_{2}-\mathrm{CH}=\mathrm{CH}_{2}\right), 4.62-4.53(\mathrm{~m}, 2 \mathrm{H}, \mathrm{Cys}(\mathrm{Me})-H$ \& Ala- $H$ ), 4.43 (m, 1H, Phe- $H$ ), 3.19 (dd, 1H, $J=14,6$ Hz, Phe- $H \mathrm{H}$ ), 3.10 (dd, $1 \mathrm{H}, J=14,8$ Hz, Phe-H $H$ ), $3.02(\mathrm{~m}, 1 \mathrm{H}, \mathrm{Cys}(\mathrm{Me})-H \mathrm{H}), 2.78(\mathrm{dd}, 1 \mathrm{H}, J=14,8 \mathrm{~Hz}, \mathrm{Cys}(\mathrm{Me})-\mathrm{H} H$ ), 2.17 (s, 3H, Cys(Me)-SCH$\left.H_{3}\right), 1.48\left(\mathrm{~d}, 3 \mathrm{H}, J=7 \mathrm{~Hz}\right.$, Ala- $\left.\mathrm{CH}_{3}\right), 1.45$ (s, 9H, $\left.\mathrm{C}\left(\mathrm{CH}_{3}\right)_{3}\right) ;{ }^{13} \mathrm{C}-\mathrm{NMR}$ 
$\left(\mathrm{CDCl}_{3}, 100.6 \mathrm{MHz}\right): \quad 172.1\left(\mathrm{CO}_{2} \mathrm{All}\right), 171.3$ (Phe-CO), 169.7 (Cys(Me)-CO), 155.8 (OCONH), 136.4 (ipso-ArC), $131.7\left(\mathrm{CH}=\mathrm{CH}_{2}\right), 129.3$ \& 128.9 ( o \& m-ArCH), 127.3 ( $p$ $\operatorname{ArCH}), 118.8\left(\mathrm{CH}=\mathrm{CH}_{2}\right), 80.8\left(C\left(\mathrm{CH}_{3}\right)_{3}\right), 66.1\left(\mathrm{CH}_{2}-\mathrm{CH}=\mathrm{CH}_{2}\right), 56.1$ (Phe- $\left.\mathrm{C}\right), 52.0$ (Cys(Me)-C ), 48.6 (Ala- $C$ ), 38.0 (Phe- $C$ ), 36.1 (Cys(Me)-C ), $\left.28.4\left(\mathrm{C}_{(\mathrm{CH}}\right)_{3}\right), 18.1$ (Ala- $\left.\mathrm{CH}_{3}\right), 15.9$ (Cys(Me)-SCH$)_{3}$; ESMS: $m / z: 494.3(\mathrm{M}+\mathrm{H})^{+}, 516.3(\mathrm{M}+\mathrm{Na})^{+}$; HRMS $\left(\mathrm{ES}^{+}\right)$: calcd for $\mathrm{C}_{24} \mathrm{H}_{35} \mathrm{~N}_{3} \mathrm{O}_{6} \mathrm{SNa}(\mathrm{M}+\mathrm{Na})^{+}, 516.2139$, found 516.2136; HPLC (ELS): 11.2 min (conditions 1 ).

Boc-Phe-Cys $(\mathrm{Me})-\operatorname{Ser}\left({ }^{t} \mathrm{Bu}\right)-\mathrm{O}^{t} B u(4 d)$. NMM (0.27 mL, $2.49 \mathrm{mmol}, 2$ equiv) was dissolved in dry $\mathrm{CH}_{2} \mathrm{Cl}_{2}(14 \mathrm{~mL})$. $\mathrm{H}-\mathrm{Ser}\left({ }^{t} \mathrm{Bu}\right)-\mathrm{O}^{t} \mathrm{Bu} \mathrm{HCl}(0.475 \mathrm{~g}, 1.87 \mathrm{mmol}, 1.5$ equiv) in dry $\mathrm{CH}_{2} \mathrm{Cl}_{2}(28 \mathrm{~mL})$ was then added followed by Boc-Phe-Cys(Me)-OH (0.477 g, 1.25 mmol, 1 equiv) and HOBt (0.202 g, $1.5 \mathrm{mmol}, 1.2$ equiv). The mixture was then cooled to $0 \mathrm{C}$ and $\mathrm{CMC}(0.793 \mathrm{~g}, 1.87 \mathrm{mmol}, 1.5$ equiv) added. The mixture was allowed to warm up to room temperature and left stirring for $3 \mathrm{hrs}$. It was then diluted with $\mathrm{CH}_{2} \mathrm{Cl}_{2}$ (17 mL), washed with sat. $\mathrm{NaHCO}_{3}(20 \mathrm{~mL}), 1 \mathrm{M} \mathrm{HCl}\left(10 \mathrm{~mL}\right.$ x 3), aqueous $\mathrm{NaHCO}_{3}$ $(5 \%, 10 \mathrm{~mL} \times 3)$, brine $(10 \mathrm{~mL} \times 2)$, dried $\left(\mathrm{Na}_{2} \mathrm{SO}_{4}\right)$, filtered and the solvent removed in vacuo. A slightly yellow powder $(0.58 \mathrm{~g}, 80 \%)$ was obtained which did not need further purification. $R_{f}: 0.52$ (Et $2 \mathrm{O} / 30 \%$ hexanes); mp: (52-62) C; IR (neat): $\max : 3278,1736$, 1687, $1642 \mathrm{~cm}^{-1} ;{ }^{1} \mathrm{H}-\mathrm{NMR}\left(\mathrm{CDCl}_{3}, 400 \mathrm{MHz}\right):$ 7.31-7.17 (m, 5H, Phe-ArH), 7.14 (d, $1 \mathrm{H}, J=8 \mathrm{~Hz}, \mathrm{NH}), 6.98(\mathrm{~d}, 1 \mathrm{H}, J=7 \mathrm{~Hz}, \mathrm{~N} H), 4.96(\mathrm{~d}, 1 \mathrm{H}, J=7 \mathrm{~Hz}$, Phe-NH), 4.54$4.46\left(\mathrm{~m}, 2 \mathrm{H}, \mathrm{Cys}(\mathrm{Me})-H \quad \& \operatorname{Ser}\left({ }^{t} \mathrm{Bu}\right)-H\right)$, 4.46-4.32 (m, $1 \mathrm{H}$, Phe- $H$ ), $3.78(\mathrm{dd}, 1 \mathrm{H}, J=$ 9, $\left.3 \mathrm{~Hz}, \operatorname{Ser}\left({ }^{t} \mathrm{Bu}\right)-H \mathrm{H}\right), 3.53\left(\mathrm{dd}, 1 \mathrm{H}, J=9,3 \mathrm{~Hz}, \operatorname{Ser}\left({ }^{t} \mathrm{Bu}\right)-\mathrm{H} H\right.$ ), $3.12(\mathrm{dd}, 1 \mathrm{H}, J=14,6$ Hz, Phe- $H \mathrm{H}$ ), 3.08 (dd, 1H, $J=14,6 \mathrm{~Hz}$, Phe-HH ), 2.88 (dd, 1H, $J=14,5 \mathrm{~Hz}$, Cys(Me)-HH ), 2.77 (dd, 1H, $J=14,8 \mathrm{~Hz}, \mathrm{Cys}(\mathrm{Me})-\mathrm{H} H$ ), 2.17 (s, 3H, Cys(Me)-SCH ), 1.46 (s, 9H, $\left.\mathrm{C}\left(\mathrm{CH}_{3}\right)_{3}\right), 1.38$ (s, 9H, $\left.\mathrm{C}\left(\mathrm{CH}_{3}\right)_{3}\right), 1.15$ (s, 9H, $\left.\mathrm{C}\left(\mathrm{CH}_{3}\right)_{3}\right) ;{ }^{13} \mathrm{C}-\mathrm{NMR}\left(\mathrm{CDCl}_{3}\right.$, 100.6 MHz): 171.3 (Phe-CONH), $169.7 \& 169.0$ (Cys(Me)-CONH \& Ser('Bu)-CONH), 155.5 (OCONH), 136.6 (ipso-ArC), $129.5 \& 128.8$ ( o \& m-ArCH), 127.1 (p-ArCH), 82.1, 80.4, $73.3\left(C\left(\mathrm{CH}_{3}\right)_{3}\right), 62.0\left(\operatorname{Ser}\left({ }^{t} \mathrm{Bu}\right)-C\right), 55.8(\mathrm{Phe}-C), 53.8\left(\mathrm{Ser}\left({ }^{t} \mathrm{Bu}\right)-C\right), 52.2$ (Cys(Me)-C ), 38.4 (Phe- $C$ ), $\left.36.6(\mathrm{Cys}(\mathrm{Me})-C), 28.4,28.1,27.5\left(\mathrm{C}_{(\mathrm{CH}}\right)_{3}\right), 15.9$ $\left(\mathrm{Cys}(\mathrm{Me})-\mathrm{SCH}_{3}\right)$; ESMS: $m / z: 582.3(\mathrm{M}+\mathrm{H})^{+}, 604.3(\mathrm{M}+\mathrm{Na})^{+}$; HRMS $\left(\mathrm{ES}^{+}\right)$: calcd for 
$\mathrm{C}_{29} \mathrm{H}_{47} \mathrm{~N}_{3} \mathrm{O}_{7} \mathrm{SNa}(\mathrm{M}+\mathrm{Na})^{+}$, 604.3027, found 604.3031; HPLC (ELS): $13.3 \mathrm{~min}$ (conditions 1); Anal. Calcd for $\mathrm{C}_{29} \mathrm{H}_{47} \mathrm{~N}_{3} \mathrm{O}_{7} \mathrm{~S}$ : C, 59.87; H, 8.14; N, 7.22; S, 5.51. Found: C, 59.60; H, 8.34; N, 6.93; S, 5.21.

\section{General procedure for the oxidation of thioethers}

Without $\mathrm{Sc}(\mathrm{OTf})_{3}$

To the thioether ( $0.1 \mathrm{mmol}, 1$ equiv) dissolved in $\mathrm{CH}_{2} \mathrm{Cl}_{2} / 10 \% \mathrm{EtOH}(1 \mathrm{~mL}), 60 \% \mathrm{H}_{2} \mathrm{O}_{2}$ (5.7 or 23.8 L, 1.2 or 5 equiv) was added under stirring. (Tables 1 and 2 show conversions of thioethers to sulfoxides under uncatalyzed conditions).

Table 1. Oxidation of sulfides 1a-d in the absence of $\mathrm{Sc}(\mathrm{OTf})_{3}$

\begin{tabular}{cccc}
\hline thioether & $\begin{array}{c}60 \% \mathrm{H}_{2} \mathrm{O}_{2} \\
\text { (equiv) }\end{array}$ & time (h) & $\begin{array}{c}\text { Yield }^{a}(\% \\
\text { sulfoxide) }\end{array}$ \\
\hline 1a & 1.2 & 24 & 43 \\
$\mathbf{1 a}$ & 5 & 24 & 76 \\
$\mathbf{1 b}$ & 1.2 & 24 & 39 \\
$\mathbf{1 b}$ & 5 & 24 & 53 \\
$\mathbf{1 c}$ & 1.2 & 24 & 76 \\
$\mathbf{1 c}$ & 5 & 20 & $>98$ \\
$\mathbf{1 d}$ & 1.2 & 24 & $29^{b}$ \\
$\mathbf{1 d}$ & 5 & 24 & $60^{c}$ \\
\hline${ }^{a}$ Determined by ${ }^{\mathrm{H}} \mathrm{H}$ NMR $;{ }^{b} 0.4 \%$ of sulfone formed; ${ }^{c} 2 \%$ of sulfone formed.
\end{tabular}

Table 1. Oxidation of thioethers 4a-d in the absence of $\mathrm{Sc}(\mathrm{OTf})_{3}$

\begin{tabular}{cccc}
\hline thioether & $\begin{array}{c}60 \% \mathrm{H}_{2} \mathrm{O}_{2} \\
\text { (equiv) }\end{array}$ & time (h) & $\begin{array}{c}\text { Yield }^{a, b}(\% \\
\text { sulfoxide) }\end{array}$ \\
\hline $\mathbf{4 a}$ & 1.2 & 6 & 15 \\
$\mathbf{4 a}$ & 5 & 5 & 66 \\
$\mathbf{4 b}$ & 1.2 & 7.25 & 71 \\
$\mathbf{4 b}$ & 5 & 1.5 & 85 \\
$\mathbf{4 c}$ & 1.2 & 6 & 91 \\
$\mathbf{4 c}$ & 5 & 2 & 97
\end{tabular}




$\begin{array}{cccc}\mathbf{4 d} & 1.2 & 11 & 87 \\ \mathbf{4 d} & 5 & 2 & 91 \\ { }^{a} \text { Determined by RP-HPLC (ELS). }{ }^{b} \text { No sulfone was observed. } & \end{array}$

With $\mathrm{Sc}(\mathrm{OTf})_{3}$ (Phenyl prenyl sulfide)

$\mathrm{Sc}(\mathrm{OTf})_{3}\left(9.8 \mathrm{mg}, 0.02 \mathrm{mmol}, 0.2\right.$ equiv) was suspended in $\mathrm{CH}_{2} \mathrm{Cl}_{2} / 10 \% \mathrm{EtOH}(0.4 \mathrm{~mL})$ and $60 \% \mathrm{H}_{2} \mathrm{O}_{2}$ (5.7 or 23.8 L, 1.2 or 5 equiv) was added under stirring. After 1 minute the substrate was also added (17.8 $\mathrm{mg}, 0.1 \mathrm{mmol}, 1$ equiv) dissolved in $\mathrm{CH}_{2} \mathrm{Cl}_{2} / 10 \% \mathrm{EtOH}(0.3 \mathrm{~mL})$. The vial in which the substrate was weighed was further washed with the solvent mixture $(0.3 \mathrm{~mL})$ and added to the reaction mixture.

With $\mathrm{Sc}(\mathrm{OTf})_{3}$ (all other thioethers)

$\mathrm{Sc}(\mathrm{OTf})_{3}\left(9.8 \mathrm{mg}, 0.02 \mathrm{mmol}, 0.2\right.$ equiv) was suspended in $\mathrm{CH}_{2} \mathrm{Cl}_{2} / 10 \% \mathrm{EtOH}(1 \mathrm{~mL})$ and $60 \% \mathrm{H}_{2} \mathrm{O}_{2}$ (5.7 or 23.8 L, 1.2 or 5 equiv) was added under stirring. After 5 minutes the substrate was also added ( $0.1 \mathrm{mmol}, 1$ equiv).

\section{Quantification of conversions.}

Oxidations were monitored by TLC and HPLC. Conversions were evaluated via ${ }^{1} \mathrm{H}-\mathrm{NMR}$ $\left(\mathrm{CDCl}_{3}\right)$ and HPLC (ELS detector).

\section{Work-up procedure.}

The reaction mixture was diluted with $\mathrm{CH}_{2} \mathrm{Cl}_{2}$, poured in a separating funnel containing water and extracted. The organic phase was washed again with water, dried $\left(\mathrm{MgSO}_{4}\right)$ and the solvent removed in vacuo.

Phenyl prenyl sulfoxide (2a). ${ }^{12}$ A liquid was obtained (96\%) which did not need further purification. $R_{f}$ : 0.41 ( $\mathrm{CHCl}_{3} / 10 \%$ EtOAc); IR (neat): 1044 (SO st) $\mathrm{cm}^{-1} ;{ }^{1} \mathrm{H}-\mathrm{NMR}$ $\left(\mathrm{CDCl}_{3}, 400 \mathrm{MHz}\right): \quad 7.62-7.41(\mathrm{~m}, 5 \mathrm{H}), 5.07$ (tt, $\left.1 \mathrm{H}, J=8,2 \mathrm{~Hz}\right), 3.56(\mathrm{dd}, 1 \mathrm{H}, J=13$, $8 \mathrm{~Hz}), 3.50(\mathrm{dd}, 1 \mathrm{H}, J=13,8 \mathrm{~Hz}), 1.71(\mathrm{~s}, 3 \mathrm{H}), 1.41(\mathrm{~s}, 3 \mathrm{H}) ;{ }^{13} \mathrm{C}-\mathrm{NMR}\left(\mathrm{CDCl}_{3}, 100.6\right.$ MHz): $143.7,142.3,136.6,131.1,129.0,124.5,111.2,56.8,26.0,18.1$; ESMS: $m / z$ : $195(\mathrm{M}+\mathrm{H})^{+}, 217(\mathrm{M}+\mathrm{Na})^{+}, 236\left(\mathrm{M}+\mathrm{H}+\mathrm{CH}_{3} \mathrm{CN}\right)^{+}, 258\left(\mathrm{M}+\mathrm{Na}+\mathrm{CH}_{3} \mathrm{CN}\right)^{+}, 389(2 \mathrm{M}+\mathrm{H})^{+}$, $411(2 \mathrm{M}+\mathrm{Na})^{+}, 452\left(2 \mathrm{M}+\mathrm{Na}+\mathrm{CH}_{3} \mathrm{CN}\right)^{+} ; \operatorname{HPLC}(254): 9.2 \mathrm{~min}$ (conditions 1).

$p$-Bromophenyl methyl sulfoxide $(2 b) .{ }^{13}$ An off-white solid was obtained (95\%) which did not need further purification. IR (neat): 1048 (SO st) $\mathrm{cm}^{-1} ;{ }^{1} \mathrm{H}-\mathrm{NMR}\left(\mathrm{CDCl}_{3}, 400 \mathrm{MHz}\right)$ : 
$7.68(\mathrm{~m}, 2 \mathrm{H}), 7.53(\mathrm{~m}, 2 \mathrm{H}), 2.72\left(\mathrm{~s}, 3 \mathrm{H}, \mathrm{CH}_{3}\right) ;{ }^{13} \mathrm{C}-\mathrm{NMR}\left(\mathrm{CDCl}_{3}, 100.6 \mathrm{MHz}\right): \quad$ 145.0, 132.7, 125.5, 125.2, 44.1; GC-CIMS: rt: $12.29 \mathrm{~min} ; m / z: 219+221(\mathrm{M})^{+} ; \operatorname{HPLC}\left({ }_{254}\right)$ : 3.0 min (conditions 2).

p-Methoxyphenyl methyl sulfoxide $(2 c)^{14}$. The residue was purified by column chromatography (silica gel, hexanes/EtOAc 6/4) to give a clear liquid (97\%). IR (neat): 1045 (SO st) $\mathrm{cm}^{-1} ;{ }^{1} \mathrm{H}-\mathrm{NMR}\left(\mathrm{CDCl}_{3}, 400 \mathrm{MHz}\right): \quad 7.58(\mathrm{~d}, J=9 \mathrm{~Hz}, 2 \mathrm{H}), 7.02$ (d, $J=9$ $\mathrm{Hz}, 2 \mathrm{H}), 3.84$ (s, 3H), 2.68 (s, 3H); ${ }^{13} \mathrm{C}-\mathrm{NMR}\left(\mathrm{CDCl}_{3}, 100.6 \mathrm{MHz}\right): \quad 162.1,136.8$, 125.5, 115.0, 55.6, 44.1; GC-CIMS: rt: $12.09 \mathrm{~min} ; \mathrm{m} / z: 171(\mathrm{M}+\mathrm{H})^{+}$.

Phenyl methyl sulfoxide $(2 d) .{ }^{4}$ The crude liquid was purified by column chromatography (silica gel, $\mathrm{CHCl}_{3}$ ) to give a liquid (61\%). $R_{f}: 0.17\left(\mathrm{CHCl}_{3}\right)$; IR (neat): ${ }_{\text {max }}: 1041$ (SO st) $\mathrm{cm}^{-1}$; ${ }^{1} \mathrm{H}-\mathrm{NMR}\left(\mathrm{CDCl}_{3}, 300 \mathrm{MHz}\right)$ : 7.68-7.60 (m, 2H, Ar-CH), 7.66-7.45 (m, 2H, Ar$\mathrm{CH}$ ), 2.71 (s, 3H, $\left.\mathrm{CH}_{3}\right) ;{ }^{13} \mathrm{C}-\mathrm{NMR}\left(\mathrm{CDCl}_{3}, 75.5 \mathrm{MHz}\right): 145.7$ (ipso- $\left.\mathrm{ArC}\right), 131.1,129.4$, $123.5(\mathrm{ArCH}), 44.0\left(\mathrm{CH}_{3}\right)$; GC-CIMS: rt $7.01 \mathrm{mins}, \mathrm{m} / z: 141(\mathrm{M}+\mathrm{H})^{+}, 158\left(\mathrm{M}+\mathrm{NH}_{4}\right)^{+}$; GC: $7.01 \mathrm{~min}$; HPLC (Daicel OD-H, UV detector ( 254 ), flow rate $0.5 \mathrm{~mL} \mathrm{~min}{ }^{-1}$, hexane/2-propanol 9/1): $23.74+26.93 \mathrm{~min}$.

Fmoc-Cys $((\mathrm{O}) \mathrm{Me})$-OMe (5a). The crude was purified by column chromatography (silica gel, EtOAc) to give a white solid (91\%). $R_{f}: 0.40$ (chloroform/10\%MeOH); mp: (152153) C; IR (neat): $\quad$ max: 3302, 1738, 1692, 1083, $1020 \mathrm{~cm}^{-1} ;{ }^{1} \mathrm{H}-\mathrm{NMR}\left(\mathrm{CDCl}_{3}, 400\right.$ MHz): $7.77 \& 7.61\left(2 \mathrm{~d}, J=7 \mathrm{~Hz}, 4 \mathrm{H}\right.$, Fmoc- $\left.\mathrm{H}_{1,4,5,8}\right) .7 .41 \& 7.33(2 \mathrm{t}, J=7 \mathrm{~Hz}, 4 \mathrm{H}$, Fmoc- $\left.\mathrm{H}_{2,3,6,7}\right), 6.09-5.95(\mathrm{~m}, 1 \mathrm{H}, \mathrm{N} H)$, 4.85-4.67 (m, 1H, Cys((O)Me)-H ), 4.52-4.34 (m, $\left.2 \mathrm{H}, \mathrm{Fmoc}-\mathrm{CH}_{2}\right), 4.30-4.19$ (m, 1H, Fmoc-CH), $3.83 \& 3.82\left(2 \mathrm{~s}, 3 \mathrm{H}, \mathrm{OCH}_{3}\right), 3.42-3.15$ $\left(\mathrm{m}, 2 \mathrm{H}, \mathrm{Cys}((\mathrm{O}) \mathrm{Me})-\mathrm{H}_{2}\right), 2.68-2.67\left(2 \mathrm{~s}, 3 \mathrm{H}, \mathrm{Cys}((\mathrm{O}) \mathrm{Me})-\mathrm{SCH}_{3}\right) ;{ }^{13} \mathrm{C}-\mathrm{NMR}\left(\mathrm{CDCl}_{3}\right.$, $100.6 \mathrm{MHz}): \quad 170.5 \& 170.3\left(\mathrm{CO}_{2} \mathrm{Me}\right), 156.1 \& 155.9(\mathrm{OCONH}), 143.8 \& 143.7$ $\left(\right.$ Fmoc- $\left.C_{8 \mathrm{a}, 9 \mathrm{a}}\right), 141.48 \& 141.47$ (Fmoc- $C_{4 \mathrm{a}, 4 \mathrm{~b}}$ ), $128.0 \& 127.9$ (2Fmoc- $\left.C_{1,8}\right), 127.3$ (Fmoc- $\left.C_{4,5}\right), 125.3\left(\right.$ Fmoc- $\left.C_{2,7}\right), 120.18 \& 120.16\left(\right.$ Fmoc- $\left.C_{3,6}\right), 67.5\left(\right.$ Fmoc- $\left.\mathrm{CH}_{2}\right), 56.0 \&$ $55.1(\mathrm{Cys}((\mathrm{O}) \mathrm{Me})-C), 53.3 \& 53.2\left(\mathrm{OCH}_{3}\right), 50.8 \& 50.3(\mathrm{Cys}((\mathrm{O}) \mathrm{Me})-C), 47.2(\mathrm{Fmoc}-$ $\mathrm{CH}), 39.5 \& 39.2\left(\mathrm{Cys}((\mathrm{O}) \mathrm{Me})-\mathrm{SCH}_{3}\right)$; ESMS: $m / z: 388.2(\mathrm{M}+\mathrm{H})^{+}, 410.1(\mathrm{M}+\mathrm{Na})^{+}$, $775.3(2 \mathrm{M}+\mathrm{H})^{+}, 797.3(2 \mathrm{M}+\mathrm{Na})^{+}$; HRMS $\left(\mathrm{ES}^{+}\right)$: calcd for $\mathrm{C}_{20} \mathrm{H}_{21} \mathrm{NO}_{5} \mathrm{SNa}(\mathrm{M}+\mathrm{Na})^{+}$, 410.10327, found 410.10326; HPLC ( 254 ): 9.5 min (conditions 1). 
Boc-Phe-Cys((O)Me)-Cys(Trt)-OMe (5b). A white foam (96\%) was obtained which did not need further purification. $R_{f}: 0.43\left(\mathrm{CHCl}_{3} / 3 \% \mathrm{MeOH}\right)$; mp: (68-75) C; IR (neat): $\max$ : 3282, 1744, 1656, $1021 \mathrm{~cm}^{-1}$; ${ }^{1} \mathrm{H}-\mathrm{NMR}\left(\mathrm{CDCl}_{3}, 400 \mathrm{MHz}\right): 8.16(\mathrm{~d}, 1 \mathrm{H}, J=8 \mathrm{~Hz}$, Cys(Trt)-NH), 7.76 (d, 1H, $J=8$ Hz, Cys(Trt)-NH), 7.75-7.63 (br s, 1H, Cys((O)Me)$\mathrm{NH}$ ), 7.43-7.12 (m, 40H, ArH), 4.96 (br s, 2H, Phe-NH), 4.92-4.80 (m, 2H, Cys((O)Me)$H$ ), 4.41-4.26 (m, 2H, Phe- $H$ ), 4.25-4.16 (m, 2H, Cys(Trt)- $H$ ), $3.6435 \& 3.6334$ (s, 6H, $\left.\mathrm{CO}_{2} \mathrm{CH}_{3}\right), 3.36(\mathrm{dd}, 1 \mathrm{H}, J=14,8 \mathrm{~Hz}, \mathrm{Cys}((\mathrm{O}) \mathrm{Me})-H \mathrm{H}), 3.23-2.90(\mathrm{~m}, 5 \mathrm{H}, 3 \mathrm{Phe}-\mathrm{HH} \&$ $\left.2 \mathrm{Cys}((\mathrm{O}) \mathrm{Me})-H_{2}\right), 2.84$ (s, 3H, Cys $\left.((\mathrm{O}) \mathrm{Me})-\mathrm{SCH}_{3}\right), 2.85-2.74(\mathrm{~m}, 2 \mathrm{H}, \mathrm{Phe}-\mathrm{HH} \&$ $\mathrm{Cys}((\mathrm{O}) \mathrm{Me})-\mathrm{H} H$ ), 2.70-2.53 (m, 4H, Cys(Trt)- $\mathrm{H}_{2}$ ), 2.60 (s, 3H, Cys((O)Me)-SCH$H_{3}$, $1.38 \& 1.37\left(2 \mathrm{~s}, 18 \mathrm{H}, \mathrm{C}\left(\mathrm{CH}_{3}\right)_{3}\right) ;{ }^{13} \mathrm{C}-\mathrm{NMR}\left(\mathrm{CDCl}_{3}, 100.6 \mathrm{MHz}\right): \quad 171.7,171.3,170.5$, 169.7, 169.1, $169.0\left(2 \mathrm{CO}_{2} \mathrm{Me} \& 4 \mathrm{CONH}\right), 155.8 \& 155.6(\mathrm{OCONH}), 144.5 \& 144.4$ (Cys(Trt)-ipso-ArC), 136.5 \& 136.4 (Phe-ipso-ArC), 129.8, 129.7, 129.3, 128.9, 128.1, 128.0, 127.9, 127.8, 127.7, 127.4, 127.3, 127.2, $126.9(\mathrm{ArCH}), 80.7\left(\mathrm{C}_{\left.\left(\mathrm{CH}_{3}\right)_{3}\right), 67.3}\right.$ (Cys(Trt)-Ar $\left.{ }_{3} C\right), 56.5(\mathrm{Phe}-\mathrm{C}), 55.0(\mathrm{Cys}((\mathrm{O}) \mathrm{Me})-\mathrm{C}), 52.7\left(\mathrm{OCH}_{3}\right), 52.6\left(\mathrm{OCH}_{3}\right), 52.5$ (Cys(Trt)-C ), $51.7(\mathrm{Cys}((\mathrm{O}) \mathrm{Me})-C), 50.1(\mathrm{Cys}((\mathrm{O}) \mathrm{Me})-C), 47.2(\mathrm{Cys}((\mathrm{O}) \mathrm{Me})-C), 39.5$ (Cys((O)Me)-SCH$\left.{ }_{3}\right), 38.0$ (Phe-C ), 37.8 (Cys((O)Me)-SCH$\left.H_{3}\right), 33.3$ (Cys(Trt)-C ), 33.2

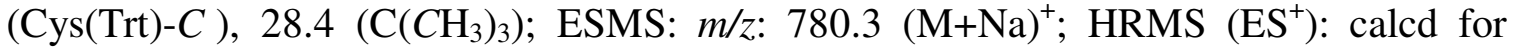
$\mathrm{C}_{41} \mathrm{H}_{48} \mathrm{~N}_{3} \mathrm{O}_{7} \mathrm{~S}_{2}(\mathrm{M}+\mathrm{H})^{+}, 758.2928$, found 758.2922; calcd for $\mathrm{C}_{41} \mathrm{H}_{47} \mathrm{~N}_{3} \mathrm{O}_{7} \mathrm{~S}_{2} \mathrm{Na}(\mathrm{M}+\mathrm{Na})^{+}$, 780.2747, found 780.2731; calcd for $\mathrm{C}_{41} \mathrm{H}_{47} \mathrm{~N}_{3} \mathrm{O}_{7} \mathrm{~S}_{2} \mathrm{~K}(\mathrm{M}+\mathrm{K})^{+}$, 796.2487, found 796.2469; HPLC (ELS): 13.0 min (conditions 1).

Boc-Phe-Cys((O)Me)-Ala-OAll (5c). A white solid was obtained which was purified by precipitation from $\mathrm{CH}_{2} \mathrm{Cl}_{2} /$ hexanes (92\%). $R_{f}$ : $0.44\left(\mathrm{CHCl}_{3} / 1 \% \mathrm{MeOH}\right) ; \mathrm{mp}$ : (115-120) C; IR (neat): max : 3288, 1739, 1689, 1645, 1024 (SO st) cm ${ }^{-1} ;{ }^{1} \mathrm{H}-\mathrm{NMR}\left(\mathrm{CDCl}_{3}, 400 \mathrm{MHz}\right)$ : 8. 24 (d, 1H, $J=8 \mathrm{~Hz}$, Ala-NH), 8.01-7.84 (br s, 1H, Cys $\left.^{2}((\mathrm{O}) \mathrm{Me})-\mathrm{N} H\right), 7.86$ (d, 1H, $J$ $=7 \mathrm{~Hz}$, Ala-NH), $7.45\left(\mathrm{~d}, 1 \mathrm{H}, J=6 \mathrm{~Hz}, \mathrm{Cys}^{1}((\mathrm{O}) \mathrm{Me})-\mathrm{N} H\right), 7.36-7.13(\mathrm{~m}, 10 \mathrm{H}$, Phe$\mathrm{ArH}), 5.90\left(\mathrm{~m}, 2 \mathrm{H}, \mathrm{CH}=\mathrm{CH}_{2}\right), 5.35(\mathrm{~m}, 1 \mathrm{H}, \mathrm{CH}=\mathrm{CHH}), 5.31(\mathrm{~m}, 1 \mathrm{H}, \mathrm{CH}=\mathrm{C} H \mathrm{H}), 5.26$ $(\mathrm{m}, 1 \mathrm{H}, \mathrm{CH}=\mathrm{CH} H), 5.23(\mathrm{~m}, 1 \mathrm{H}, \mathrm{CH}=\mathrm{CH} H), 5.10-4.91(\mathrm{~m}, 3 \mathrm{H}, 2 \mathrm{Boc}-\mathrm{N} H \&$ $\left.\mathrm{Cys}^{2}((\mathrm{O}) \mathrm{Me})-H\right), 4.87$ (td, $\left.1 \mathrm{H}, J=7,3 \mathrm{~Hz}, \mathrm{Cys}^{1}((\mathrm{O}) \mathrm{Me})-H\right), 4.65-4.59\left(\mathrm{~m}, 4 \mathrm{H}, \mathrm{CH}_{2^{-}}\right.$ $\left.\mathrm{CH}=\mathrm{CH}_{2}\right), 4.57-4.42(\mathrm{~m}, 2 \mathrm{H}$, Ala- $H$ ), 4.41-4.27 (m, 2H, Phe- $H$ ), $3.46(\mathrm{dd}, 1 \mathrm{H}, J=14,8$ 
$\left.\mathrm{Hz}, \mathrm{Cys}^{1}((\mathrm{O}) \mathrm{Me})-H \mathrm{H}\right), 3.32-3.22\left(\mathrm{~m}, 1 \mathrm{H}, \mathrm{Cys}^{2}((\mathrm{O}) \mathrm{Me})-H \mathrm{H}\right), 3.19(\mathrm{dd}, 1 \mathrm{H}, J=14,5$ Hz, Phe- $H \mathrm{H}$ ), 3.13 (dd, 1H, $J=14,6 \mathrm{~Hz}$, Phe-HH ), 3.08-2.90 (m, 2H, Cys ${ }^{2}((\mathrm{O}) \mathrm{Me})-$ $\mathrm{H} H$ \& Phe- $H \mathrm{H}$ ), 2.96 (dd, $1 \mathrm{H}, J=14,9 \mathrm{~Hz}$, Phe-HH ), 2.86 (s, 3H, Cys((O)Me)-CH , $_{3}$, $2.85\left(\mathrm{dd}, 1 \mathrm{H}, J=14,3 \mathrm{~Hz}, \mathrm{Cys}^{1}((\mathrm{O}) \mathrm{Me})-\mathrm{H} H\right), 2.63$ (s, 3H, Cys $\left.((\mathrm{O}) \mathrm{Me})-\mathrm{CH}_{3}\right), 1.45$ (d, $\left.\left.3 \mathrm{H}, J=8 \mathrm{~Hz}, \mathrm{Ala}-\mathrm{CH}_{3}\right), 1.43(\mathrm{~d}, 3 \mathrm{H}, J=7 \mathrm{~Hz} \text {, Ala-CH})_{3}\right), 1.393\left(\mathrm{~s}, 9 \mathrm{H}, \mathrm{C}\left(\mathrm{CH}_{3}\right)_{3}\right), 1.386$ (s, 9H, $\left.\mathrm{C}\left(\mathrm{CH}_{3}\right)_{3}\right) ;{ }^{13} \mathrm{C}-\mathrm{NMR}\left(\mathrm{CDCl}_{3}, 100.6 \mathrm{MHz}\right): \quad 172.1,172.0,171.7,171.3\left(\mathrm{CO}_{2} \mathrm{All}\right.$ \& Phe-CONH), $169.0 \& 168.9$ (Cys((O)Me)-CO), $156.0 \& 155.5$ (OCONH), 136.4 (ipso$\mathrm{ArC}), 131.9 \& 131.8\left(\mathrm{CH}=\mathrm{CH}_{2}\right), 129.32,129.28,128.94,128.86$ ( o \& $\left.m-\mathrm{ArCH}\right), 127.2$ $(p-\mathrm{ArCH}), 118.8 \& 118.6\left(\mathrm{CH}=\mathrm{CH}_{2}\right), 80.8 \& 80.6\left(C\left(\mathrm{CH}_{3}\right)_{3}\right), 66.0 \& 65.9\left(\mathrm{CH}_{2^{-}}\right.$ $\left.\mathrm{CH}=\mathrm{CH}_{2}\right), 56.4 \& 56.0\left(\right.$ Phe- $C$ ), $54.9\left(\mathrm{Cys}^{2}((\mathrm{O}) \mathrm{Me})-C\right), 51.7\left(\mathrm{Cys}^{1}((\mathrm{O}) \mathrm{Me})-C\right), 50.4$ $\left(\operatorname{Cys}^{2}((\mathrm{O}) \mathrm{Me})-C\right), 48.7(\mathrm{Ala}-\mathrm{C}), 47.2\left(\mathrm{Cys}^{1}((\mathrm{O}) \mathrm{Me})-C\right), 39.6\left(\mathrm{Cys}((\mathrm{O}) \mathrm{Me})-\mathrm{CH}_{3}\right), 38.1$ (Phe- $C$ ), $\left.37.7\left(\mathrm{Cys}((\mathrm{O}) \mathrm{Me})-\mathrm{CH}_{3}\right), 28.4\left(\mathrm{C}_{(\mathrm{CH}}\right)_{3}\right), 17.50$ \& $17.35\left(\right.$ Ala- $\left.\mathrm{CH}_{3}\right)$; ESMS: m/z: $510.1(\mathrm{M}+\mathrm{H})^{+}, 1018.9(2 \mathrm{M}+\mathrm{H})^{+}$; HRMS $\left(\mathrm{ES}^{+}\right)$: calcd for $\mathrm{C}_{24} \mathrm{H}_{35} \mathrm{~N}_{3} \mathrm{O}_{7} \mathrm{SNa}(\mathrm{M}+\mathrm{Na})^{+}$, 532.2088, found 532.2080; HPLC (ELS): 9.6 min (conditions 1).

Boc-Phe-Cys $((O) M e)-\operatorname{Ser}\left({ }^{t} B u\right)-O^{t} B u(5 d)$. A white solid (92\%) was obtained which did not need further purification. $R_{f}: 0.50\left(\mathrm{CHCl}_{3} / 1 \% \mathrm{MeOH}\right)$; mp: (58-65) C; IR (neat): max: 3282, 1735, 1710, 1681, 1647, 1020 (SO st) cm ${ }^{-1}$; ${ }^{1} \mathrm{H}-\mathrm{NMR}\left(\mathrm{CDCl}_{3}, 400 \mathrm{MHz}\right): \quad 7.99$ $\left(\mathrm{d}, 1 \mathrm{H}, J=8 \mathrm{~Hz}, \operatorname{Ser}\left({ }^{t} \mathrm{Bu}\right)-\mathrm{N} H\right), 7.62\left(\mathrm{~d}, 1 \mathrm{H}, J=7 \mathrm{~Hz}, \mathrm{Cys}^{2}((\mathrm{O}) \mathrm{Me})-\mathrm{N} H\right), 7.59(\mathrm{~d}, 1 \mathrm{H}, J$ $\left.=9 \mathrm{~Hz}, \operatorname{Ser}\left({ }^{t} \mathrm{Bu}\right)-\mathrm{N} H\right), 7.37\left(\mathrm{~d}, 1 \mathrm{H}, J=7 \mathrm{~Hz}, \mathrm{Cys}^{1}((\mathrm{O}) \mathrm{Me})-\mathrm{N} H\right), 7.33-7.14(\mathrm{~m}, 10 \mathrm{H}$, $\operatorname{Ar} H)$, 5.04-4.86 (m, 4H, Phe-NH \& $\left.\mathrm{Cys}^{2}((\mathrm{O}) \mathrm{Me})-H \& \mathrm{Cys}^{1}((\mathrm{O}) \mathrm{Me})-H\right), 4.49(\mathrm{~m}, 2 \mathrm{H}$, $\operatorname{Ser}\left({ }^{t} \mathrm{Bu}\right)-H$ ), 4.48-4.32 (m, 2H, Phe- $H$ ), 3.77 (dd, $\left.2 \mathrm{H}, J=9,4 \mathrm{~Hz}, \operatorname{Ser}\left({ }^{t} \mathrm{Bu}\right)-H_{2}\right), 3.54$ (dd, $1 \mathrm{H}, J=10,3 \mathrm{~Hz}, \operatorname{Ser}\left({ }^{t} \mathrm{Bu}\right)-H \mathrm{H}$ ), 3.52 (dd, $1 \mathrm{H}, J=10,3 \mathrm{~Hz}, \operatorname{Ser}\left({ }^{t} \mathrm{Bu}\right)-\mathrm{H} H$ ), 3.45 (dd, $\left.1 \mathrm{H}, J=14,7 \mathrm{~Hz}, \mathrm{Cys}^{1}((\mathrm{O}) \mathrm{Me})-H \mathrm{H}\right), 3.22-3.03$ (m, 4H, Phe- $\left.H_{2}, \mathrm{Cys}^{2}((\mathrm{O}) \mathrm{Me})-H_{2}\right)$, 3.11-2.92 (m, 2H, Phe- $\left.H_{2}\right), 2.85$ (dd, $\left.1 \mathrm{H}, J=14,7 \mathrm{~Hz}, \mathrm{Cys}^{1}((\mathrm{O}) \mathrm{Me})-\mathrm{H} H\right), 2.84$ (s, 3H, $\left.\mathrm{Cys}((\mathrm{O}) \mathrm{Me})-\mathrm{SCH}_{3}\right), 2.63$ (s, 3H, Cys((O)Me)-SCH$\left.H_{3}\right), 1.47$ (s, 18H, C(CH $\left.)_{3}\right), 1.38$ (s, 18H, $\left.\mathrm{C}\left(\mathrm{CH}_{3}\right)_{3}\right), 1.16$ (s, $\left.18 \mathrm{H}, \mathrm{C}\left(\mathrm{CH}_{3}\right)_{3}\right) ;{ }^{13} \mathrm{C}-\mathrm{NMR}\left(\mathrm{CDCl}_{3}, 100.6 \mathrm{MHz}\right): \quad 171.7,171.2$, 169.0, 168.97, $168.92\left(\mathrm{CO}_{2}{ }^{t} \mathrm{Bu} \&\right.$ Phe-CONH \& $\left.\mathrm{Cys}((\mathrm{O}) \mathrm{Me})-C \mathrm{ONH}\right), 155.4(\mathrm{OCONH})$, 136.6 \& 136.5 (ipso-ArC), 129.5, 129.4, 128.8 ( o \& m-ArCH), $127.15 \& 127.10$ ( $p$ $\operatorname{ArCH}), 81.95,81.92,80.4,73.34,73.31\left(C\left(\mathrm{CH}_{3}\right)_{3}\right), 61.81 \& 61.75\left(\operatorname{Ser}\left({ }^{t} \mathrm{Bu}\right)-C\right), 55.9$ 
(Phe-C ), $55.4\left(\mathrm{Cys}^{2}((\mathrm{O}) \mathrm{Me})-C\right), 54.1 \& 54.0\left(\mathrm{Ser}\left({ }^{t} \mathrm{Bu}\right)-C\right), 52.8\left(\mathrm{Cys}^{1}((\mathrm{O}) \mathrm{Me})-C\right)$, $49.7 \& 47.1\left(\mathrm{Cys}^{1}((\mathrm{O}) \mathrm{Me})-C \& \mathrm{Cys}^{2}((\mathrm{O}) \mathrm{Me})-C\right), 39.6\left(\mathrm{Cys}((\mathrm{O}) \mathrm{Me})-\mathrm{SCH}_{3}\right), 38.3$ (Phe$C), 38.2\left(\mathrm{Cys}((\mathrm{O}) \mathrm{Me})-\mathrm{SCH}_{3}\right), 28.4,28.2,27.4\left(\mathrm{C}_{\left.\left(\mathrm{CH}_{3}\right)_{3}\right)}\right)$ ESMS: $\mathrm{m} / \mathrm{z}: 620.2(\mathrm{M}+\mathrm{Na})^{+}$; HRMS $\left(\mathrm{ES}^{+}\right)$: calcd for $\mathrm{C}_{29} \mathrm{H}_{47} \mathrm{~N}_{3} \mathrm{O}_{8} \mathrm{SNa}(\mathrm{M}+\mathrm{Na})^{+}, 620.2976$, found 620.2974 ; HPLC (ELS): 11.6 min (conditions 1)

\section{Solid phase synthesis}

Fmoc-Cys(Me)-OH was loaded on HMPA-PS resin (1\% DVB) (HMPA linker was synthesised according to literature protocol ${ }^{15}$ and loaded onto amino methyl polystyrene resin using standard ester bond formation conditons) using standard solid-phase synthesis protocols. ${ }^{16}$ The loading was evaluated by Fmoc test (experimental $0.80 \mathrm{mmolg}^{-1}$, calcd $\left.0.89 \mathrm{mmolg}^{-1}\right)$.

Treatment of polymer supported Fmoc-Cys(Me)-OH with $\mathrm{H}_{2} \mathrm{O}_{2}$ and catalytic $\mathbf{S c}(\mathbf{O T f})_{3}$. $\mathrm{Sc}(\mathrm{OTf})_{3}(9.8 \mathrm{mg}, 0.02 \mathrm{mmol}, 0.2$ equiv) was suspended under stirring in $\mathrm{CH}_{2} \mathrm{Cl}_{2} / 10 \% \mathrm{EtOH}(0.4 \mathrm{~mL})$ in HPLC vials. $60 \% \mathrm{H}_{2} \mathrm{O}_{2}(21.3 \mathrm{~L}, 0.5 \mathrm{mmol}$, 5equiv) was then added. After 1 minute, the mixture was added to the pre-swollen (in $\mathrm{CH}_{2} \mathrm{Cl}_{2} / 10 \% \mathrm{EtOH}$ ) resin (100mg, $0.089 \mathrm{mmol}$ based on calcd loading). The vial was then washed with $\mathrm{CH}_{2} \mathrm{Cl}_{2} / 10 \% \mathrm{EtOH}(2 \times 0.3 \mathrm{~mL})$ and the washings added to the resin. The mixture was shaken in a rotatory wheel for $10 \mathrm{~min}$. The resin was washed with $\mathrm{CH}_{2} \mathrm{Cl}_{2} / 10 \% \mathrm{EtOH}(3 \times 1 \mathrm{~mL}), \mathrm{CH}_{2} \mathrm{Cl}_{2}(3 \times 1 \mathrm{~mL}), \mathrm{MeOH}(3 \times 1 \mathrm{~mL}), \mathrm{Et}_{2} \mathrm{O}(3 \times 1 \mathrm{~mL})$ and dried. The resin was then swollen again in $\mathrm{CH}_{2} \mathrm{Cl}_{2}$ before treatment with TFA/ $\mathrm{CH}_{2} \mathrm{Cl}_{2} /$ water $(95 / 3 / 2,1 \mathrm{~mL})$ for $1.5 \mathrm{hr}$. Resin was washed with the cleavage cocktail $(4 \times 0.5 \mathrm{~mL})$. The combined cleavage and washings solutions were evaporated to dryness and the residue analysed by RP-HPLC comparing the retention times with known samples of 6 and 7 the preparation of which is detailed below.

Fmoc-Cys $((\mathrm{O}) \mathrm{Me})-\mathrm{OH}(6) .{ }^{17}$ Fmoc-Cys(Me)-OH (100 mg, $0.28 \mathrm{mmol}, 1$ equiv) was dissolved in $\mathrm{MeOH}(2.5 \mathrm{~mL})$ and the resulting solution cooled in an ice bath. Sodium periodate $(65.8 \mathrm{mg}, 0.31 \mathrm{mmol}, 1.1$ equiv) in water $(1 \mathrm{~mL})$ was then added dropwise and the resulting mixture allowed to warm up to room temperature and left stirring for 1.5 hrs. After this time, the $\mathrm{MeOH}$ was evaporated and the aqueous mixture transferred into a separating funnel containing brine acidified at $\mathrm{pH}=1(10 \mathrm{~mL})$. The mixture was extracted with EtOAc $(5 \times 40 \mathrm{~mL}$ ) (problems were encountered to the the low solubility 
of the product in most organic solvents, large volumes of solvents were therefore used). The organic phases were then put together, dried $\left(\mathrm{Na}_{2} \mathrm{SO}_{4}\right)$, filtered and the solvent removed in vacuo. The resulting powder was triturated with $\mathrm{EtOH}(15 \mathrm{~mL}), \mathrm{Et}_{2} \mathrm{O}$ (15 $\mathrm{mL})$ and dried (99 mg, 95\%). $R_{f}: 0.37\left(9 \mathrm{CH}_{2} \mathrm{Cl}_{2}: 1 \mathrm{MeOH}: 0.1 \mathrm{AcOH}\right) ; \mathrm{mp}$ : higher than $230 \mathrm{C}$; IR (neat): $\max : 3327,1733,1688,1044$ (SO st) cm ${ }^{-1} ;{ }^{1} \mathrm{H}-\mathrm{NMR}$ (DMSO-d 6400 MHz): $\quad 8.01 \& 7.94(2 \mathrm{~d}, 2 \mathrm{H}, J=8 \mathrm{~Hz}, \mathrm{NH}), 7.89 \& 7.71(2 \mathrm{~d}, 8 \mathrm{H}, J=7 \mathrm{~Hz}$, Fmoc$\left.\mathrm{H}_{1,4,5,8}\right), 7.41 \& 7.33\left(2 \mathrm{t}, 8 \mathrm{H}, J=7 \mathrm{~Hz}\right.$, Fmoc-H $\left.\mathrm{H}_{2,3,6,7}\right), 4.50-4.16\left(\mathrm{~m}, 8 \mathrm{H}\right.$, Fmoc-CH $\mathrm{CH}_{2} \& H$ \& Fmoc-CH), $3.25(\mathrm{dd}, 1 \mathrm{H}, J=13,6 \mathrm{~Hz}, H), 3.15-2.96(\mathrm{~m}, 3 \mathrm{H}, H), 2.505 \& 2.500(2 \mathrm{~s}$, $\left.6 \mathrm{H}, \mathrm{CH}_{3}\right) ;{ }^{13} \mathrm{C}-\mathrm{NMR}\left(\mathrm{DMSO}-\mathrm{d}_{6}, 100.6 \mathrm{MHz}\right): \quad 172.3 \& 171.9\left(\mathrm{CO}_{2} \mathrm{H}\right), 156.0 \& 155.8$ (OCONH), 143.8 (Fmoc-C $8 \mathrm{a}, 9 \mathrm{a}), 140.8$ (Fmoc- $\left.C_{4 \mathrm{a}, 4 \mathrm{~b}}\right), 127.7$ (Fmoc- $C_{1,8}$ ), 127.2 (Fmoc$\left.C_{4,5}\right), 125.3\left(\right.$ Fmoc- $\left.C_{2,7}\right), 120.2\left(\right.$ Fmoc- $\left.C_{3,6}\right), 65.8\left(\right.$ Fmoc- $\left.\mathrm{CH}_{2}\right), 54.2(C), 54.1(C), 48.8$ (C), $48.1(\mathrm{C}), 46.6$ (Fmoc- $\mathrm{CH}), 38.5\left(\mathrm{CH}_{3}\right), 38.2\left(\mathrm{CH}_{3}\right)$; HRMS $\left(\mathrm{ES}^{+}\right)$: calcd for $\mathrm{C}_{19} \mathrm{H}_{19} \mathrm{~N}_{1} \mathrm{O}_{5} \mathrm{~S}_{1} \mathrm{Na}(\mathrm{M}+\mathrm{Na})^{+}, 396.0882$, found 396.0876; HPLC (ELS): 8.8 min (conditions 1)

Fmoc-Cys $\left(\left(\mathrm{O}_{2}\right) \mathrm{Me}\right)-\mathrm{OH}$ (7). Fmoc-Cys(Me)-OH (100 mg, $0.28 \mathrm{mmol}, 1$ equiv) was dissolved in $\mathrm{MeOH}(2.5 \mathrm{~mL})$ and the resulting solution cooled in an ice bath. OXONE (516 mg, $0.84 \mathrm{mmol}, 3$ equiv) in water $(1 \mathrm{~mL})$ was then added dropwise and the resulting mixture allowed to warm up to rt and left stirring for $1.5 \mathrm{hrs}$. After this time, the $\mathrm{MeOH}$ was evaporated and the aqueous mixture transferred into a separating funnel containing brine acidified to $\mathrm{pH}=1(10 \mathrm{~mL})$. The mixture was extracted with EtOAc $(2 \times 5 \mathrm{~mL})$. The organic phases were then put together, dried $\left(\mathrm{Na}_{2} \mathrm{SO}_{4}\right)$, filtered and the solvent removed in vacuo to give a white powder (105 mg, 96\%) which did not need further purification. $R_{f}: 0.52\left(\mathrm{CH}_{2} \mathrm{Cl}_{2}: \mathrm{MeOH}: \mathrm{AcOH}\right.$ 9:1:0.1); mp: (185-190) C; IR (neat): $\max$ : 3302, 1742, 1701, $1313\left(\mathrm{SO}_{2}\right.$ st as), $1124\left(\mathrm{SO}_{2}\right.$ st s) $\mathrm{cm}^{-1} ;{ }^{1} \mathrm{H}-\mathrm{NMR}\left(\mathrm{DMSO}_{6}, 400\right.$ MHz): $7.93(\mathrm{~d}, 1 \mathrm{H}, J=9 \mathrm{~Hz}, \mathrm{NH}), 7.89 \& 7.70\left(2 \mathrm{~d}, 4 \mathrm{H}, J=7 \mathrm{~Hz}, \mathrm{Fmoc}-\mathrm{H}_{1,4,5,8}\right), 7.42$ \& $7.32\left(2 \mathrm{t}, 4 \mathrm{H}, J=7 \mathrm{~Hz}\right.$, Fmoc-H $\left.{ }_{2,3,6,7}\right), 4.46(\mathrm{~m}, 1 \mathrm{H}, H$ ), 4.39-4.27 (m, 2H, Fmoc-CH , , $4.24\left(\mathrm{t}, 1 \mathrm{H}, J=7 \mathrm{~Hz}\right.$, Fmoc-CH), 3.60-3.46 (m, 2H, $H$ ), $2.96\left(\mathrm{~s}, 3 \mathrm{H}, \mathrm{CH}_{3}\right) ;{ }^{13} \mathrm{C}-\mathrm{NMR}$ (DMSO-d 6 , $100.6 \mathrm{MHz}): \quad 171.1\left(\mathrm{CO}_{2} \mathrm{H}\right), 155.7(\mathrm{OCONH}), 143.7\left(\right.$ Fmoc- $\left.C_{8 \mathrm{a}, 9 \mathrm{a}}\right), 140.7$ (Fmoc- $C_{4 \mathrm{a}, 4 \mathrm{~b}}$ ), 127.6 (Fmoc- $\left.C_{1,8}\right), 127.1$ (Fmoc- $\left.C_{4,5}\right), 125.2$ (Fmoc- $C_{2,7}$ ), 120.1 (Fmoc- 
$\left.C_{3,6}\right), 65.8\left(\right.$ Fmoc- $\left.\mathrm{CH}_{2}\right), 54.5(\mathrm{C}), 48.8(\mathrm{C}), 49.2(\mathrm{C}), 46.6(\mathrm{Fmoc}-\mathrm{CH}), 41.5\left(\mathrm{CH}_{3}\right)$; ESMS: $m / z$ : 501.9 (M+TFA-H) ${ }^{-}$HRMS $\left(\mathrm{ES}^{+}\right)$: calcd for $\mathrm{C}_{19} \mathrm{H}_{19} \mathrm{NO}_{5} \mathrm{SNa}(\mathrm{M}+\mathrm{Na})^{+}$, 412.0825, found 412.0840; HPLC (ELS): 9.8 min (conditions 1).

\section{References}

(1) Nilsson, Y. I. M.; Andersson, P. G.; Bäckvall, J-E. J. Am. Chem. Soc. 1993, 115, 6609-6613.

(2) (a) Buchanan, G. W.; Reyes-Zamora, C.; Clarke, D. E. Can. J. Chem. 1974, 52, 3895 3904. (b) Jiang, X-K.; Ji, G-Z. J. Org. Chem. 1992, 57, 6051-6056.

(3) Ali, M. H.; Bohnert, G. J. Synth. Commun. 1998, 28, 2983-2998.

(4) Sato, K.; Hyodo, M.; Aoki, M.; Zheng, X-Q.; Noyori, R. Tetrahedron 2001, 57, 24692476.

(5) Cheng, D.; Zhu, S.; Yu, Z.; Cohen, T. J. Am. Chem. Soc. 2001, 123, 30-34.

(6) Burrage, S.; Bradley, M. Ph.D. Dissertation, University of Southampton, UK, 1998.

(7) Rachele, J. R. J. Org. Chem. 1963, 28, 2898.

(8) Desimoni, G.; Dusi, G.; Faita, G.; Quadrelli, P.; Righetti, P. Tetrahedron, 1995, 51, 4131-4144.

(9) Rudolph, J.; Theis, H.; Hanke, R.; Endermann, R.; Johannsen, L.; Geschke, F-U. J. Med. Chem. 2001, 44, 619-626.

(10) (a) Hoover, D. J.; Wester, R. T.; Rosati, R. L. Eur. Pat. Appl. 1988 EP 266950. (b) Luly, J. R.; BaMaung, N.; Soderquist, J.; Fung, A. K. L.; Stein, H. D.; Kleinert, H. D.; Marcotte, P. A.; Egan, D. A.; Bopp, B.; Merits, I.; Bolis, G.; Greer, J.; Perun, T. J.; Plattner, J. J. J. Med. Chem. 1988, 31, 2264-2276.

(11) Waldmann, H.; Kunz, H. Liebigs Ann. Chem. 1983, 1712-1725.

(12) Uneyama, K.; Hasegawa, N.; Kawafuchi, H., Torii, S. Bull. Chem. Soc. Jpn. 1983, 56, 1214-1218.

(13) Cooke, R. S.; Hammond, G. S. J. Am. Chem. Soc. 1970, 92, 2739-2745.

(14) Ali, M. H.; Stevens, W. C. Synthesis 1997, 764-768.

(15) Sheppard, R. C.; Williams, B. J. Int. J. Peptide Protein Res. 1982, 20, 451-454.

(16) Burrage, S.; Raynham, T.; Williams, G.; Essex, J. W.; Allen, C.; Cardno, M.; Swali, V.; Bradley, M. Chem. Eur. J. 2000, 6, 1455-1466. 